\title{
Comparison of Entropy vs. Bispectral Index Anesthetic Depth Monitoring during Isoflurane Anesthesia in Living Donor Liver Transplantation: Observational Study
}

\author{
Sang Hyun Lee' ${ }^{(D}$, Janie Jang1, Kyunga Kim² ${ }^{(D}$, Heejin Yoo $^{2} \mathbb{C}^{(}$, \\ Yu Jeong Bang1 ${ }^{(\mathbb{D}}$, Ji-Won Choi ${ }^{1}$, Gaab Soo Kim ${ }^{*}{ }^{*}$ \\ ${ }^{1}$ Department of Anesthesiology and Pain Medicine, Samsung Medical Center, Sungkyunkwan University School of Medicine, \\ Seoul, Korea \\ ${ }^{2}$ Statistics and Data Center, Research Institute for Future Medicine, Samsung Medical Center, Seoul, Korea \\ Email: ^gskim@skku.edu
}

How to cite this paper: Lee, S.H., Jang, J., Kim, K., Yoo, H., Bang, Y.J., Choi, J.-W. and Kim, G.S. (2021) Comparison of Entropy vs. Bispectral Index Anesthetic Depth Monitoring during Isoflurane Anesthesia in Living Donor Liver Transplantation: Observational Study. Open Journal of Anesthesiology, 11, 137-148.

https://doi.org/10.4236/ojanes.2021.115013

Received: April 6, 2021

Accepted: May 9, 2021

Published: May 12, 2021

Copyright $\odot 2021$ by author(s) and Scientific Research Publishing Inc. This work is licensed under the Creative Commons Attribution International License (CC BY 4.0).

http://creativecommons.org/licenses/by/4.0/

\begin{abstract}
Liver transplantation (LT) recipients are known to require less anesthetic agents. Providing minimally required anesthetics while avoiding awareness is especially important in LT recipients because it would help manage perioperative hemodynamic instability with less vasopressor and fast track recovery. This study aims to compare state entropy (SE) against bispectral index (BIS) during isoflurane anesthesia in LT. We adjusted anesthesia to BIS values 40 60 , and compared it with concomitant SE values. BIS, SE values, and anesthetic requirements according to liver failure severity, etiology and LT stages were analyzed. For BIS-SE differences, SE value that is different from the concomitant BIS by more than 15 was defined as a significant disagreement. Mann Whitney, Kruskal Wallis test and a Poisson exact test were used for analysis. The BIS-SE pair sets of 2895 from 38 patients were analyzed. BIS, SE values and anesthetic requirements were significantly lower in MELD $\geq 20$ ( $p$ $<0.001$ in all) and in alcoholic etiology ( $p<0.001$ in all). For BIS-SE differences, 320 disagreement data pairs were seen at a rate of 1.33 times/hr $(95 \%$ $\mathrm{CI}=[1.19,1.48], \mathrm{p}<0.001)$. A significant disagreement was delineated in MELD score $\geq 20$ (3.04 times/hr, CI $=[2.64,3.49], \mathrm{p}<0.001)$, alcoholic etiology $(3.19$ times/hr, $[2.67,3.78], \mathrm{p}<0.001)$ and postreperfusion stage $(1.63$ times/hr, $[1.43,1.85], \mathrm{p}<0.001)$. In these significant BIS-SE differences, 95.9\% (307/320 disagreement data pairs) showed higher BIS than SE. In conclusion, in high MELD and alcoholic etiology, anesthetic requirements were significantly less, and BIS and SE showed great discrepancy with lower SE values. Therefore, when SE monitoring is used during LT, anesthesiologists
\end{abstract}


may need to consider that in high MELD and alcoholic etiology, SE tends to show lower values than the concomitant BIS values that are within optimal anesthetic depth ranges.

\section{Keywords}

Anesthetic Depth, Bispectral Index, Entropy, Liver Transplantation, Isoflurane

\section{Introduction}

Defining a proper anesthetic depth in patients with end stage liver diseases (ESLD) is an important concern to anesthesiologists caring for recipients of liver transplantation (LT). These patients are known to show decreased sensitivity to pain and reduced requirement for anesthetics, partly because of increased endogenous opioid peptides and pathophysiology of ESLD [1] [2] [3] [4]. Therefore, providing minimally required anesthetics can prevent too profound anesthesia and help manage hemodynamic instability with a decreased need for vasopressors [5]. It is crucial among many other anesthetic concerns because LT often accompanies hemodynamic instability owing to physiology of hyper-dynamic circulation, bleeding and surgical procedures [6] [7].

Among a few literature, one previous study from our institution showed that LT recipients with higher model for end-stage liver disease (MELD) score showed less desflurane inhalational anesthetic requirements during preanhepatic and anhepatic stages [4]. In our practice, we use the Bispectral index ${ }^{\mathrm{TM}}$ (BIS ${ }^{\mathrm{TM}}$, Medtronic Covidien, Dubline, Ireland) as our standard anesthetic depth monitoring. But in some institutions, Entropy ${ }^{\mathrm{TM}}$ (GE Healthcare, Helsinki, Finland) is also a commonly used frontal electroencephalogram.

As there lacks information on the use of Entropy in anesthetic depth management in LT recipients, we designed this observational study to investigate BIS and state entropy (SE) values per se, and compare the SE module against the BIS reference values according to liver diseases severity, etiology and LT stages during isoflurane anesthesia for living donor liver transplantation (LDLT). Anesthetic requirements in liver diseases severity, etiology and LT stages were also investigated.

\section{Patients and Methods}

\subsection{Ethics Approval}

This observational study was approved by the institution board of our hospital, Samsung Medical Center institutional review board on May 42016 (IRB no. SMC 2016-04-106).

The registration number to the Clinical Research Information Service (CRIS, https://cris.nih.go.kr/cris/index/index.do) is KCT0002339. This study was performed in observation of all the relevant guidelines and regulations. 


\subsection{Patient Enrollment}

Patients aged between 20 and 69 years and undergoing elective LDLT were consecutively recruited during the period from June 2016 through May 2017. Informed consents were received from all patients. All consecutive elective LT recipients anesthetized by the hospital's uniform protocol and monitored by both BIS and entropy were enrolled. Exclusion criteria were previous history or current status with hepatic encephalopathy, intraoperative opioids use and preoperatively hemodynamically unstable patients.

\subsection{Anesthetic Management}

Patients did not receive any premedication. Standard patient monitoring including electrocardiography (EKG), noninvasive blood pressure (NIBP), peripheral oxygen saturation, inspired faction and end tidal concentration of sevoflurane, isoflurane, oxygen and carbon dioxide were applied (Datax Ohmeda $S / 5^{\mathrm{mm}}$ Anesthesia Monitor, Datex-Ohmeda, Helsinki, Finland). Anesthesia was induced with thiopental sodium $5 \mathrm{mg} / \mathrm{kg}$. After loss of consciousness, vecuronium 1 $\mathrm{mg} / \mathrm{kg}$ or rocuronium $0.8 \mathrm{mg} / \mathrm{kg}$ was given intravenously for muscle relaxation. Tracheal intubation was carried out after full relaxation. Immediately after the end of anesthetic induction and after wiping the patient's forehead with an alcohol pad and drying, BIS sensor (BIS Quarto sensor, Medtronic-Covidien, Dublin, Ireland) and Entropy sensor (GE Healthcare, Helsinki, Finland) were placed on the forehead according to the manufacturers' recommendation. Hemodynamic profiles were monitored with radial and femoral arterial blood pressure, femoral venous pressure, central venous pressure and pulmonary artery pressure (PAP) via Swan Ganz catheterization. Blood pressure, heart rate, cardiac output (CO), right ventricular ejection fraction (RV EF) and systemic venous resistance (SVR) were referenced for hemodynamic management. Dopamine or norepinephrine was continuously infused if $\mathrm{RV} \mathrm{EF}<40 \%$ or SVR $<600$ dyn $\cdot \sec / \mathrm{cm}^{5}$. Anesthesia was maintained with sevoflurane at the surgical incision and changed to isoflurane after surgical retractor application. We did not use opioids for routine anesthetic management, but if arterial systolic blood pressure persisted above $160 \mathrm{mmHg}$ despite inhalational anesthetic increment, remifentanil continuous infusion was used. If remifentanil was used intraoperatively, the patient data were excluded from the final analysis. A continuous infusion of muscle relaxant was achieved with atracurium at $5-10 \mathrm{mcg} / \mathrm{kg} / \mathrm{min}$. At reperfusion, atropine $0.5 \mathrm{mg}$ or epinephrine $20 \mathrm{mcg}$ or calcium chloride $300 \mathrm{mg}$ was given intravenously at the discretion of anesthesiologist. Hemostatic management was carried out using platelet count, prothrombine time expressed as international normalized ratio (INR), serum fibrinogen, thromboelastography (TEG) and clinical estimation of bleeding in the surgical field.

\subsection{Data Collection}

The BIS and SE values (BIS-SE pairs) were collected every five minutes throughout 
isoflurane maintenance and were collected up to 4 hours after reperfusion. Isoflurane concentration was adjusted to achieve BIS values $40-60$, and concomitant SE entropy values were observed. BIS ranges of $<40$ or $>60$ lasting less than 5 minues were tolerated in between isoflurane adjustment because of clinical insignificance. The patients' liver diseases severity was categorized by MELD score $<20$ vs. $\geq 20$ [4] [8]. The MELD score is a reliable model to predict short term mortality rate, with a previous study result of 3-month mortality rate of $<29 \%$ in MELD score $\leq 19$, whereas the same period mortality rate of $>76 \%$ in MELD $\geq$ 20 [8]. All data were noted of their LT stages of preanhepatic, anhepatic and postreperfusion stages. The collected data included BIS value, SQI (signal quality index), EMG (electromyogram) of BIS, SE, response entropy (RE) and end tidal isoflurane (ET-iso). All the BIS values with SQI $\geq 80$ were used or for SQI $<80$, mean for the immediately before and after data were calculated and used.

\subsection{Postoperative Assessment}

At the end of surgery, the patients were transferred to the intensive care unit (ICU). At the ICU, when the patients recovered from surgery and became able to communicate, we interviewed all patients to assess the possibility of explicit awareness during LDLT. For this purpose, we asked that patients using the modified Brice questionnaires which take about 15-20 minutes to answer [9]. The modified brice questionnaire includes the following questions: 1) What was the last thing you remember before going to sleep? 2) What is the first thing you remember after waking up? 3) Do you remember anything between going to sleep and waking up? 4) Did you dream during your procedure? 5) What was the worst thing about your operation?

\subsection{Statistics}

BIS, SE values and anesthetic requirements (ET-iso) were compared in groups according to liver diseases severity (MELD score $<20$ vs. $\geq 20$ ), etiology (alcoholic vs. hepatitis viral vs. miscellaneous) and LT stages (preanhepatic vs. anhepatic vs. postreperfusion) by using Mann Whitney U test or Kruskal Wallis test.

For overall BIS and SE correlation during LT, spearman's correlation analysis was performed.

For comparison of concomitant BIS and SE during LT, a Poisson analysis was used. We assumed that the number of disagreement events during one hour would follow as a Poisson distribution with an average event rate $(\lambda)[10]$. We hypothesized that one disagreement event can occur at most during an hour by chance (i.e., baseline average event rate $\lambda_{0} \leq 1$ ). Using a Poisson exact test, we declared the significant disagreement between the two parameters if the disagreement events occurred more than once in an hour (i.e., alternate average event rate $\lambda_{1}>1$ ). In a previous study, disagreements $\geq 10$ unit, lasting $\geq 60$ seconds between BIS and SE values, were used to analyze differences in raw EEG signals between the two [11]. In light of the reference and also based on our 
clinical experience, in our study, a significant disagreement was defined as any SE value different from the concomitant BIS value by more than 15 . The analysis of BIS-SE disagreement according to liver disease severity, etiology and LT stages were carried out using the poisson test and chi square test.

Sample size was calculated using nQuery + nTerim 4.0 software for an assumed alternate average event rate $\left(\lambda_{1}\right)$ of 1.5 with a significance level $\alpha=0.05$ and expected power $\beta=80 \%$, and the required sample size (the number of patients) was 31.

$\mathrm{P}$ values $<0.05$ were considered significant. Statistical analysis was performed using IBM SPSS ${ }^{\circledR}$ Statistics software ver. 18 (SPSS Inc. Chicago, IL, USA), SAS version 9.4 (SAS Institute Inc, Cary, NC, USA) and R 3.3.3 (Vienna, Austria; http://www.R-project.org/).

\section{Results}

Forty nine patients were screened and 45 were recruited during the period from June 2016 through May 2017 at our institution and 3330 data sets were collected. Seven of 45 patents were excluded because SQI data were not collected in the first three patients $(\# 1,2,3)$, remifenanil was used intraoperatively in two patients $(\# 28,34)$, sevoflurane was used throughout the surgery in one patient (\#37) and an entropy sensor posed error during monitoring in one patient (\#44). Finally, 38 patients with 2895 data were included in the analysis (Figure 1).

CONSORT Flow Diagram

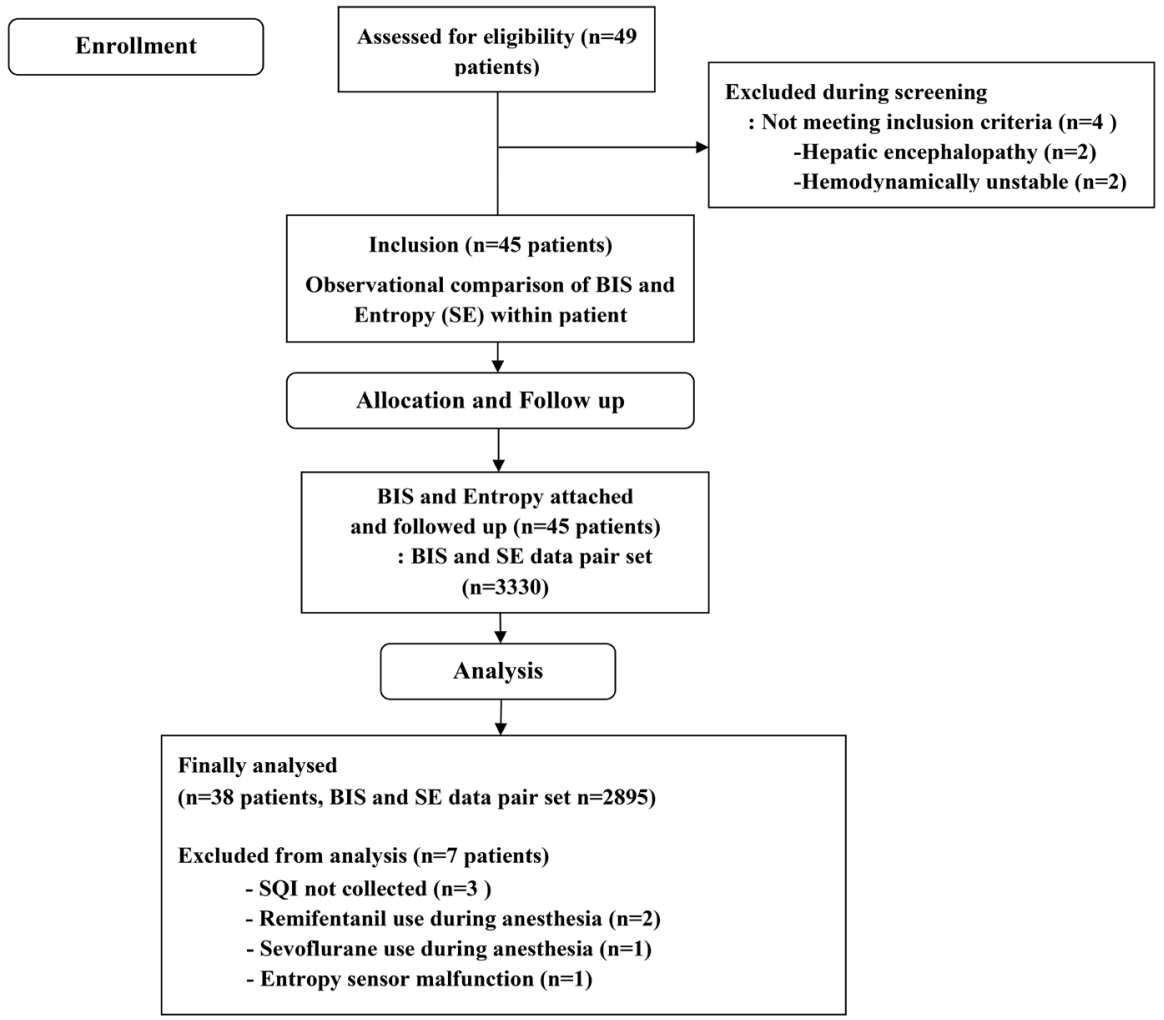

Figure 1. CONSORT flow diagram. 
Demographic and intraoperative data are displayed in Table 1.

Our patient with alcoholic etiology often presented with high MELD score (6 of 7 patients).

BIS and SE values grouped according to liver disease severity showed that BIS and SE values were significantly lower in MELD $\geq 20(\mathrm{p}<0.001, \mathrm{p}<0.001)$ compared to those in MELD $<20$. Anesthetic depth requirement, ET-iso, was also significantly lower in MELD $\geq 20$ ( $\mathrm{p}<0.001$ ). In analysis according to etiology, alcoholic etiology showed significantly lower BIS and SE values ( $\mathrm{p}<$ $0.001, \mathrm{p}<0.001)$ compared to those of other etiologies, with ET-iso also showing significantly lower anesthetic requirement in alcoholic etiology group among other etiologies ( $\mathrm{p}<0.001)$ (Table 2$)$.

Overall during LT, SE and BIS showed strong correlation $\left(\mathrm{r}_{\mathrm{s}}=0.750, \mathrm{p}<\right.$ 0.001) (Figure 2). BIS was higher than SE in 2015 (69.6\%) of 2895 data set.

However, comparison of BIS and SE showed that a significant disagreement (Figure 2, BIS and SE value differences of more than 15) was detected in 320 among 2895 BIS-SE data pairs. According to Poisson analysis, the average detection

Table 1. LT recipient demographics $(n=38)$ and intraoperative data of 2895 BIS-SE data pairs.

\begin{tabular}{|c|c|}
\hline \multicolumn{2}{|c|}{ Demographics and BIS-SE data } \\
\hline Age (year) & $55[52-60]$ \\
\hline Sex (Male/Female) (number of patients) & $32 / 6$ \\
\hline Height $(\mathrm{cm})$ & $166.9[163.0-171.6]$ \\
\hline Weight $(\mathrm{kg})$ & $68.8[57.0-74.1]$ \\
\hline \multicolumn{2}{|l|}{ Body temperature ${ }^{\circ} \mathrm{C}$} \\
\hline Intraoperative lowest/highest & $35.8[35.6-36.2] / 36.9[36.4-37.2]$ \\
\hline MELD & $12[8-20]$ \\
\hline$<20$ (number of patients) & 27 \\
\hline$\geq 20$ (number of patients) & 11 \\
\hline \multicolumn{2}{|l|}{ Etiology of hepatic failure } \\
\hline Alcoholic (number of patients) & 7 \\
\hline Hepatitis viral (number of patients) & 28 \\
\hline Miscellaneous (number of patients) & 3 \\
\hline BIS values & $48[43-55]$ \\
\hline BIS value ranges $<40$ (number of data pair) & 167 \\
\hline $40-60$ (number of data pair) & 2508 \\
\hline$<60$ (number of data pair) & 220 \\
\hline SE values & $44[35-52]$ \\
\hline RE values & $45[36-54]$ \\
\hline ET-iso & $0.7[0.5-0.8]$ \\
\hline
\end{tabular}

Data are median [interquartile ranges] or number of patients or number of data pair. MELD, Model for End-Stage Liver Disease; BIS, bispectral index; SE, state entropy; RE, reactive entropy; ET-iso, end tidal isoflurane. 
Table 2. Anesthetic requirements, BIS, SE and BIS-SE disagreement according to MELD, etiology and LT stages.

\begin{tabular}{|c|c|c|c|c|c|c|c|c|}
\hline & \multicolumn{2}{|c|}{$\begin{array}{c}\text { End tidal isoflurane } \\
\text { concentration } \\
\text { (Anesthetic requirements) }\end{array}$} & \multicolumn{2}{|c|}{ Bispectral index } & \multicolumn{2}{|c|}{ State entropy } & \multicolumn{2}{|c|}{$\begin{array}{c}\text { Significant disagreement } \\
\text { between BIS and SE by } \\
\text { more than } 15 \\
\text { Disagreement (yes/total; \%) }\end{array}$} \\
\hline & ET-iso & $P$ value & BIS & $P$ value & SE & $P$ value & $\mid$ BIS-SE $\mid>15$ & $P$ value \\
\hline \multicolumn{9}{|l|}{ MELD } \\
\hline$<20(\mathrm{n}=2094)$ & $0.8[0.6-0.9]$ & & $49.0[44.0-56.0]$ & \multirow{2}{*}{$<0.001$} & $47.0[40.0-55.0]$ & & $117 / 2094(5.6 \%)$ & \multirow{2}{*}{$<0.001$} \\
\hline$\geq 20(\mathrm{n}=801)$ & $0.4[0.3-0.5]^{\star}$ & $<0.001$ & $44.0[42.0-49.0]^{\star}$ & & $32.0[27.0-43.0]^{\star}$ & $<0.001$ & $203 / 801(25.3 \%)^{\star}$ & \\
\hline \multicolumn{9}{|l|}{ Etiology } \\
\hline Alcoholic $(\mathrm{n}=493)$ & $0.4[0.2-0.6]^{\star \dagger}$ & \multirow{3}{*}{$\begin{array}{l}<0.001 \\
<0.001\end{array}$} & $45.0[42.0-54.0]^{* \dagger}$ & \multirow{3}{*}{$\begin{array}{c}<0.001 \\
0.001\end{array}$} & $32.0[27.0-48.0]^{* \dagger}$ & \multirow{3}{*}{$\begin{array}{c}<0.001 \\
0.073\end{array}$} & $131 / 493(26.6 \%)$ & \multirow{3}{*}{$<0.001$} \\
\hline Hepatitis viral $(\mathrm{n}=2193)$ & $0.7[0.5-0.8]^{\dagger}$ & & $48.0[44.0-55.0]^{\dagger}$ & & $45.0[37.0-53.0]$ & & $169 / 2193(7.7 \%)$ & \\
\hline Miscellaneous $(\mathrm{n}=209)$ & $1.0[0.9-1.1]$ & & $50.0[44.0-58.0]$ & & $44.0[38.0-57.0]$ & & $20 / 209(9.6 \%)$ & \\
\hline \multicolumn{9}{|l|}{ LT stages } \\
\hline Preanhepatic $(n=216)$ & $0.7[0.5-0.8]$ & & $48.0[44.0-54.5]$ & & $45.0[39.0-51.5]$ & & $12 / 216(5.6 \%)$ & \\
\hline Anhepatic $(n=906)$ & $0.6[0.5-0.8]^{*}$ & 0.047 & $47.0[42.0-54.0]^{\dagger}$ & $<0.001$ & $44.0[35.0-52.0]$ & 0.144 & $67 / 906(7.4 \%)$ & $<0.001$ \\
\hline Postreperfusion $(\mathrm{n}=1773)$ & $0.7[0.5-0.9]$ & & $48.0[44.0-55.0]$ & & $44.0[34.0-53.0]$ & & $241 / 1773(13.6 \%)$ & \\
\hline
\end{tabular}

Data are median [interquartile ranges] or number of data sets. MELD: ${ }^{*} \mathrm{P}<0.05$ is statistically significant. Etiology: ${ }^{*} \mathrm{P}<0.05$ significant compared to hepatitis viral; ${ }^{\dagger} \mathrm{P}<0.05$ significant compared to miscellaneous. LT stages: ${ }^{\star} \mathrm{P}<0.05$ significant compared to preanhepatic stage; ${ }^{\dagger} \mathrm{P}<0.05$ significant compared to postreperfusion stage. MELD, Model for End-Stage Liver Disease; LT stages, liver transplantation stages; BIS, bispectral index; SE, state entropy; ET-iso, end tidal isoflurane. $|\mathrm{SE}-\mathrm{BIS}|>15$; significant disagreement between SE and BIS values.

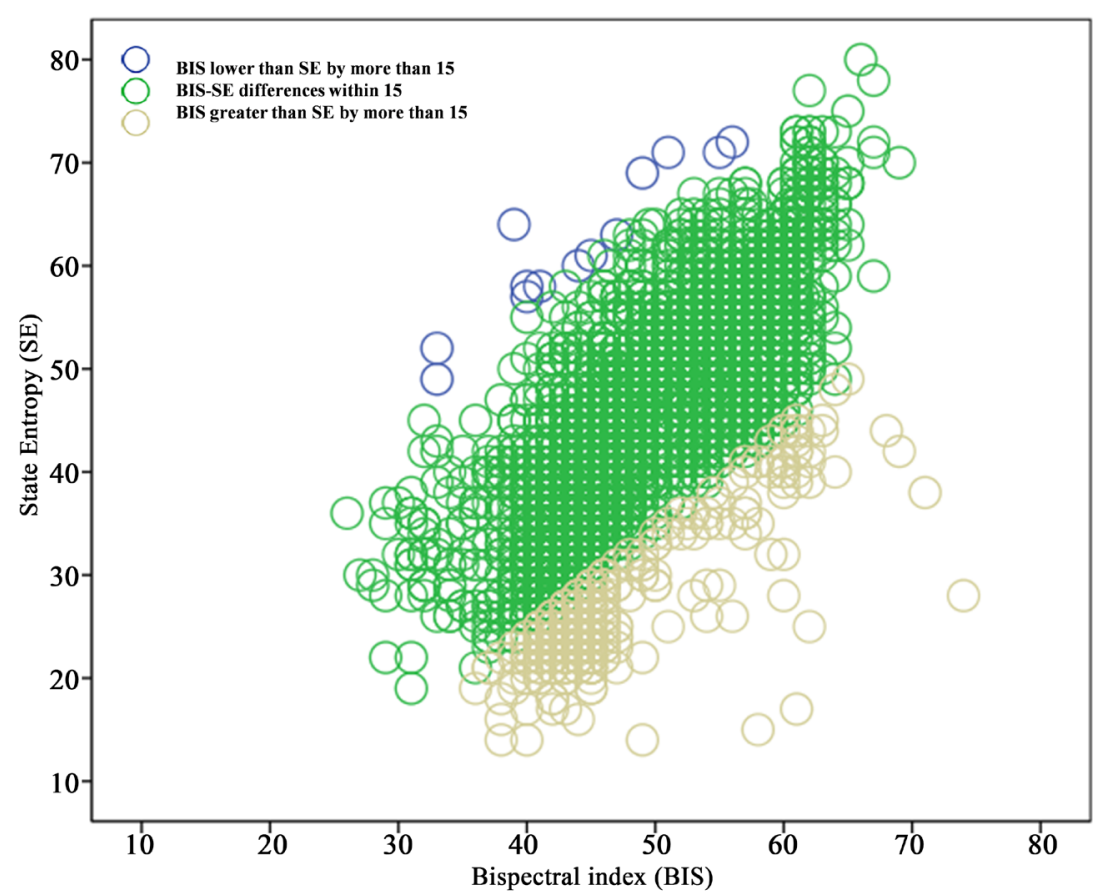

Figure 2. Scatter plot for SE and BIS.

rate of disagreement was 1.33 times/hr (95\% confidence interval $(\mathrm{CI})=[1.19$, $1.48])$, and by our definition, it indicated a significant disagreement between BIS and SE $(\mathrm{p}<0.001)$. 
Further comparison of BIS and SE according to liver diseases severity, etiology and LT stages showed that BIS and SE showed significant disagreements in MELD score $\geq 20$ (detection rate of 3.04 times/hr, 95\% CI $=[2.64,3.49], \mathrm{p}<$ $0.001)$, alcoholic etiology (3.19 times/hr, $[2.67,3.78], \mathrm{p}<0.001)$ and postreperfusion stage $(1.63$ times/hr $[1.43,1.85], \mathrm{p}<0.001)$. Table 2 and Figure 3 show significant BIS-SE differences against $\mathrm{MELD} \geq 20$, alcoholic etiology and postreperfusion stage.

In these significant BIS-SE differences, 95.9\% (307/320 disagreement data pairs) showed higher BIS than SE. Most disagreements are distributed within BIS 40 - 60 ranges (Table 3 ).
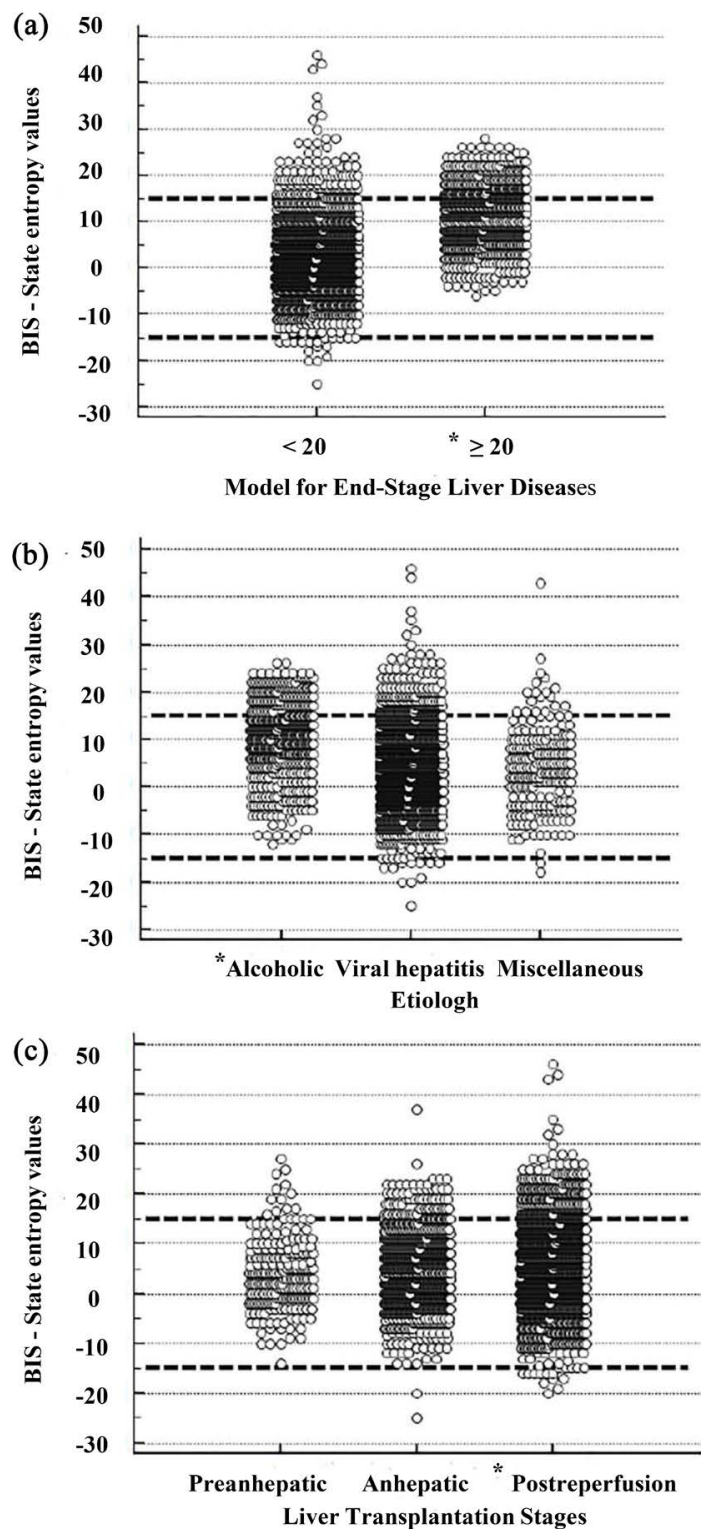

Figure 3. BIS-SE differences according to MELD, etiology and LT stages are delineated. A significant disagreement $\left({ }^{*}\right)$ was detected in (a) high MELD, (b) alcoholic etiology and (c) postreperfusion stage. Slashed bold line indicates BIS-SE differences of 15. MELD, model for end-stage liver disease; LT, liver transplantation. ${ }^{\star} \mathrm{P}<0.05$ indicates statistical significance. 
Table 3. BIS and SE disagreement data according to BIS ranges.

\begin{tabular}{cccc}
\hline & \multicolumn{3}{c}{ BIS Ranges } \\
\cline { 2 - 4 } BIS-SE data pair & $<40$ & $40-60$ & $>60$ \\
& $\mathrm{n}=167$ & $\mathrm{n}=\mathbf{2 5 0 8}$ & $\mathrm{n}=\mathbf{2 2 0}$ \\
\hline Significant disagreement data pairs $(\mathrm{n}=320 /$ total 2895) & 14 & 282 & 24 \\
BIS higher than SE ( $\mathrm{n}=307 /$ total 320 disagreement pairs $)$ & 11 & 272 & 24 \\
\hline
\end{tabular}

Data are numbers of data sets. BIS, bispectral index; SE, state entropy.

There was no explicit awareness assessed by the modified Brice questionnaire.

\section{Discussion}

We observed that while BIS and SE showed strong correlation, BIS was higher than SE in $70 \%$ of all data pairs, and if BIS-SE showed significant differences in value, BIS was higher in more than $90 \%$ of data pairs. Our observation study showed that LT recipients with MELD $\geq 20$ or alcoholic etiology required less isoflurane anesthetics to achieve anesthetic depth, but in these patients, the discrepancy between BIS and SE was significant, with SE displaying lower values than those of concomitant BIS.

The result is in line with our previous work with desflurane anesthetic agents that patients with high MELD $\geq 20$ showed less anesthetic requirement. In our study, we also found that alcoholic etiology also showed less requirement for anesthetics. Alcoholic LT recipients often come with MELD $\geq 20$, and this may explain the reason for low anesthetic requirement (ET-iso) in high MELD and alcoholic origin patients. In general, factors that increase the central nervous system (CNS) metabolic activity, neurotransmission, and CNS neurotransmitter levels increase minimal alveolar concentration (MAC). Upregulated CNS responses to chronically depressed neurotransmitter levels as in chronic alcoholism also increase MAC. However, unlike the former explanation for an increased MAC in chronic alcoholism, ESLD patients with chronic alcoholic patients with high MELD score displayed low MAC, i.e. low anesthetic requirement [12]. Moreover these patients with high MELD or alcoholic etiology displayed with significantly lower values of BIS and SE compared to those in patients with low MELD or other etiologies, respectively (Table 2). We think it is noteworthy that BIS and SE showed significant differences in these patients and SE showed lower values than those of BIS, which were within optimal anesthetic ranges. When entropy monitoring is used during LT, especially in alcoholic, high MELD patients, lower SE values with low anesthetic requirement are to be expected. In future study, comparison of non-alcoholic, high MELD patients with alcoholic, high MELD patients may differentiate whether alcoholic etiology or high MELD may be the causative reason for the low MAC.

Processed EEG monitoring to assess anesthetic depth during LT is prerequisite to deliver only the required anesthetics, reduce the possibility of hemodynamic instability caused by deep anesthesia, facilitate fast track recovery and ex- 
clude the potential chances of intraoperative explicit recall [1]. Differences in BIS and SE may be attributed to different EEG processing algorithms, or to certain EEG characteristics. In Aho et al.'s study, authors showed that SE tended to show higher value than BIS during EEG burst suppression, delta-theta dominance, and BIS tended to be higher than SE in alpha or beta activity [11] [13]. We did not investigate raw EEG patterns to find out how their EEG patterns change to anesthetics according to liver disease severity or etiology, and contribute to different values in BIS and SE. EEG studies in ESLD patients would be interesting for further study because progressive increases in neuropeptides and change in EEG patterns could explain for decreased need of anesthetics in these patients. Endogenous opioid neuropeptides which are known to increase in ESLD patients have central and peripheral analgesic effects [3] [14]. In our clinical practice, we rarely use opioids during LT and also excluded patients with intraoperative remifentanil use in this study. Therefore we assessed the pure isoflurane effect on the EEG indices.

In Table 2, anesthetic requirement was the least in anhepatic stage compared to other stages in our result. This is also compatible with a previous study by Toprak et al. which also showed that LT recipients required the least isoflurane during the anhepatic phase compared to the preanhepatic and postreperfusion stages to achieve BIS 40 - 55, although the clinical significance was minimal [15]. They used remifentanil continuous infusion $0.15 \mathrm{ug} / \mathrm{kg} / \mathrm{min}$ during surgery and ours did not [15]. In the postreperfusion stage, as the transplanted liver gradually starts to function anesthetic requirement may alter compared to preanhepatic and anhepatic stages. However, we cannot explain for the greatest discrepancy between BIS and SE during the postreperfusion stage in this study.

Limitations of this study include the following. First, the time interval for our data was 5 minutes, and we did not investigate raw EEG data or suppression ratio. An individual, continuous raw EEG data would have made analysis of different EEG wave patterns according to the liver failure severity or LT stages. This could be used to explain for differences in BIS and SE values. But the detailed EEG analysis was beyond the scope of our study, and we focused on clinical observation. Second, we did not use neuromuscular monitoring during LT. However, a continuous infusion of muscle relaxant was used in this study to maintain an adequate relaxation for the operation, therefore, EMG for BIS was uniformly less than 25 .

\section{Conclusion}

In conclusion, in high MELD and alcoholic etiology, anesthetic requirements were significantly low, and SE and BIS may show a great discrepancy with lower SE values. Therefore, when SE is used for monitoring, in high MELD score or alcoholic etiology, anesthesiologists may need to consider that SE could show lower values than the concomitant BIS values that are within optimal anesthetic depth ranges. 


\section{Conflicts of Interest}

The authors declare no conflicts of interest regarding the publication of this paper.

\section{References}

[1] Wang, C.H., Chen, C.L., Cheng, K.W., Huang, C.J., Chen, K.H., Wang, C.C., et al. (2008) Bispectral Index Monitoring in Healthy, Cirrhotic, and End-Stage Liver Disease Patients Undergoing Hepatic Operation. Transplantation Proceedings, 40, 2489-2491. https://doi.org/10.1016/j.transproceed.2008.07.031

[2] Pai, S.L., Aniskevich, S., Rodrigues, E.S. and Shine, T.S. (2015) Analgesic Considerations for Liver Transplantation Patients. Current Clinical Pharmacology, 10, 54-65. https://doi.org/10.2174/1574884709666140212101228

[3] Donovan, K.L., Janicki, P.K., Striepe, V.I., Stoica, C., Franks, W.T. and Pinson, C.W. (1997) Decreased Patient Analgesic Requirements after Liver Transplantation and Associated Neuropeptide Levels. Transplantation, 63, 1423-1429.

https://doi.org/10.1097/00007890-199705270-00010

[4] Kang, J.G., Ko, J.S., Kim, G.S., Gwak, M.S., Kim, Y.R. and Lee, S.K. (2010) The Relationship between Inhalational Anesthetic Requirements and the Severity of Liver Disease in Liver Transplant Recipients According to Three Phases of Liver transplantation. Transplantation Proceedings, 42, 854-857. https://doi.org/10.1016/j.transproceed.2010.02.057

[5] Wildes, T.S., Mickle, A.M., Ben Abdallah, A., Maybrier, H.R., Oberhaus, J., Budelier, T.P., et al. (2019) Effect of Electroencephalography-Guided Anesthetic Administration on Postoperative Delirium among Older Adults Undergoing Major Surgery: The Engages Randomized Clinical Trial. JAMA, 321, 473-483. https://doi.org/10.1001/jama.2018.22005

[6] Rahimzadeh, P., Safari, S., Faiz, S.H. and Alavian, S.M. (2014) Anesthesia for Patients with Liver Disease. Hepatitis Monthly, 14, e19881.

https://doi.org/10.5812/hepatmon.19881

[7] Adelmann, D., Kronish, K. and Ramsay, M.A. (2017) Anesthesia for Liver Transplantation. Anesthesiology Clinics, 35, 491-508. https://doi.org/10.1016/j.anclin.2017.04.006

[8] Kamath, P.S., Wiesner, R.H., Malinchoc, M., Kremers, W., Therneau, T.M., Kosberg, C.L., et al. (2001) A Model to Predict Survival in Patients with End-Stage Liver Disease. Hepatology, 33, 464-470. https://doi.org/10.1053/jhep.2001.22172

[9] Mashour, G.A. and Avidan, M.S. (2015) Intraoperative Awareness: Controversies and Non-Controversies. British Journal of Anaesthesia, 115, i20-i26. https://doi.org/10.1093/bja/aev034

[10] Ostle, B. and Malone, L. (1988) Statistics in Research: Basic Concepts and Techniques for Research Workers. Iowa State University Press, Ames, Iowa, USA.

[11] Aho, A.J., Kamata, K., Jantti, V., Kulkas, A., Hagihira, S., Huhtala, H., et al. (2015) Comparison of Bispectral Index and Entropy Values with Electroencephalogram during Surgical Anaesthesia with Sevoflurane. British Journal of Anaesthesia, 115, 258-266. https://doi.org/10.1093/bja/aev206

[12] Ebert, T. and Lindenaum, L. (2013) Inhaled Anesthetics. In: Barash, P.G., Cahalan, M.K., Cullen, B.F., Stock, M.C., Stoelting, R.K. and Ortega, R., Eds., Clinical Anesthesia, Lippincott Williams \& Wilkins, Philadelphia, PA 19103 USA, 458-459.

[13] Balci, C., Karabekir, H. and Sivaci, R. (2010) Determining Entropy Values Equiva- 
lent to the Bispectral Index Values during Sevoflurane Anaesthesia. Archives of Medical Science, 6, 370-374. https://doi.org/10.5114/aoms.2010.14257

[14] Oyama, T., Jin, T., Yamaya, R., Ling, N. and Guillemin, R. (1980) Profound Analgesic Effects of Beta-Endorphin in Man. The Lancet, 1, 122-124.

https://doi.org/10.1016/S0140-6736(80)90606-6

[15] Toprak, H.I., Sener, A., Gedik, E., Ucar, M., Karahan, K., Aydogan, M.S., et al. (2011) Bispectral Index Monitoring to Guide End-Tidal Isoflurane Concentration at Three Phases of Operation in Patients with End-Stage Liver Disease Undergoing Orthotopic Liver Transplantation. Transplantation Proceedings, 43, 892-895.

https://doi.org/10.1016/j.transproceed.2010.11.023 